\title{
Performance Analysis of Space Surveillance Using Space Based Optical Sensors
}

\author{
Federica Vitiello ${ }^{1}$ (D)
}

Received: 17 September 2020 / Revised: 17 September 2020 / Accepted: 8 October 2020 / Published online: 5 November 2020

(c) The Author(s) 2020

\begin{abstract}
This paper aims to describe the analysis of the performance of an electro-optical space-based sensor for space surveillance purposes and space debris detection in the geostationary (GEO) ring. Such sensor is considered to be operating on a dawndusk Sun-synchronous, circular low Earth orbit at an altitude of $630 \mathrm{Km}$, while its optical characteristics have been taken from those of the Space-Based Visible (SBV) sensor. Two main simulations have been carried out through the use of the MATLAB software. The first simulation deals with the detection capability of the sensor, which is discussed in terms of detectable visual magnitude when the target of the observation is a diffuse sphere orbiting in the geostationary (GEO) orbit; its minimum detectable size is then determined. In addition, the relative geometry between the Sun, the sensor and the target has also been studied along with the configurations which can limit the visibility of the sensor over the target. The second simulation has been used to evaluate the performance of the sensor in terms of number of detectable GEO targets and duration of the observation when a certain pointing strategy is adopted. In such strategy, two SBV-like sensors are placed on the same orbit, thus creating a constellation in which each sensor points towards a fixed location in the inertial space. These locations have been chosen to be the geosynchronous pinch points.
\end{abstract}

Keywords Space debris $\cdot$ Space-based optical Sensors $\cdot$ Visual magnitude $\cdot$ Solar phase angle

\section{Introduction}

The space debris population is composed of objects of various nature which have been brought in orbit by human space activities. Such objects are typically parts of bigger bodies that have detached during collisions or explosions, and their position and velocity are, in most cases, unknown. Because of the uncertainty in the pieces of information regarding this type of objects and because of their non-cooperative nature, space debris poses a serious threat to the operative space assets which are currently orbiting the Earth and to the onEarth population and activities.

The current knowledge of the resident space objects (RSO), among active satellites, inactive satellites and space debris, is mainly kept in the Satellite Catalog maintained by the US Space Surveillance Network (SSN), in which the orbital elements of the so-called catalogued objects are listed

Federica Vitiello

federitiello@gmail.com

1 Department of Industrial Engineering, University of Napoli "Federico II", Napoli, Italy at a given epoch. An explanatory example of the breadth of the space debris issue is given by the fragmentation debris population, which is composed of debris whose genesis is related to fragmentation events. As a matter of fact, as referred to the state of affairs of January the 1st of 2019, it has been found that of the more than 19000 catalogued RSOs orbiting the Earth, the fragmentation debris population accounts for around the 55\% [1]. The diameter of these objects can be as small as $1 \mu \mathrm{m}$; nevertheless, the current detection capability of the SSN sensors is set on RSOs whose diameter is larger than $10 \mathrm{~cm}$, at low Earth orbits (LEO) altitudes and larger than $80 \mathrm{~cm}$, at GEO altitudes [2]. This latter measurement is primarily obtained by optical ground-based telescopes.

It appears to be clear that in order to ensure the safety of current and future space missions while, at the same time, protecting the on-Earth activities from the possible re-entry of unknown, non-cooperative objects, an improved knowledge of the small debris population is needed. Even though the majority of space assets operates at LEO altitudes, because of the importance of the satellites which do carry out their missions in the GEO ring, this space region is 
looked at with great attention. For this very reason, it is the purpose of this work to investigate the use of a space-based optical sensor for the detection of sub-catalogue size objects orbiting in the GEO belt in order to provide additional measurements complementing the performance of the existing ground-based systems.

Due to its location above the atmosphere of the Earth, a space-based telescope is not affected by the same limitations of its ground-based counterpart. In fact, because of the effects of the sky brightness and of the meteorological conditions, the operative time of a ground-based telescope is reduced to nighttime and to those moments when the observed sky window is clear. The measurements of a space-based sensor are immune from these constrains and are, at the same time, independent from the geographical location of the sensor, thus implying that each nation could ideally create and maintain a catalogue of observed objects with no need to rely on the measurements gained by other nations for the computation of the collision probability of their space assets. However, building and testing a spacebased telescope are a great technological challenge and, although the sensor is not affected by the presence of the Earth atmosphere, it is still mainly limited by how close it can be pointed to the Sun and by the presence of the Earth in its field of view (FoV).

The characteristics of the sensor used for the development of the simulations have been taken from those of the SBV sensor which was launched on the Midcourse Space Experiment (MSX) satellite in 1996 [3]. In particular, the FoV of such sensor has been considered to be conical with an angular width of $1.4^{\circ}$ and it is placed on a circular, dawn-dusk Sun-synchronous LEO orbit at an altitude of $630 \mathrm{Km}$ and an inclination of $98^{\circ}$, thus optimizing both the power supply of the hosting satellite and the relative geometry between the Sun, the Earth, the sensor and the target.

\section{Target Detectability}

\subsection{Visual Magnitude and Solar Phase Angle}

The detectability of a target in space can be discussed in terms of its visual magnitude. In astronomy, the apparent magnitude of a celestial body, such as a star or a galaxy, is used to define its brightness as seen by an observer which is placed at a certain distance from the body itself. When such observer is able to detect light within the visible range, as it is the case for an optical telescope, then the apparent magnitude is referred to as visual magnitude. The visual magnitude is measured on a reversed logarithmic scale, meaning that the brightest the object is, the lowest its magnitude is; the Sun, for example, which is one of the brightest celestial bodies, is considered to have a visual magnitude of -26.7 .
When the visual magnitude of a debris or, more generally, of a satellite is considered, it is obviously not referred to any intrinsic brightness of the object; indeed, it is referred to its capability to reflect light. The formula that has been used for the evaluation of the visual magnitude during this work is showed below for a diffuse spherical object having a diameter equal to $\mathrm{d}[4]$.

$m_{v, \mathrm{obj}}=m_{v, \text { ref }}-2.5 \log _{10}\left[\frac{d^{2}}{R^{2}}\left(\frac{\rho_{\text {spec }}}{4}+\rho_{\text {diff }} p_{\text {diff }}(\phi)\right)\right]$

In such equation, the quantities $\rho_{\text {spec }}$ and $\rho_{\text {diff }}$ are, respectively, the specular and diffuse reflectivity components of the surface of the observed sphere, $\mathrm{R}$ is its distance, measured in $\mathrm{Km}$, from the observer (sensor), $m_{v, \text { ref }}$ is a reference value in visual magnitude which has been chosen to be that of the Sun (-26.7), and $p_{\text {diff }}$ is the phase function which depends on the solar phase angle $\phi$. An expression can be given for the phase function of a diffuse sphere as written below [4].

$p_{\text {diff }}(\phi)=\frac{2}{3 \pi}[\sin (\phi)+(\pi-\phi) \cos (\phi)]$

The solar phase angle, which is one crucial parameter for the measurement of the visual magnitude, is an information on the illumination condition of an object in space. In fact, it is defined as the angle between the direction to the Sun and the direction to the observer as seen at the object which is being observed [5]. A more favourable illumination condition is obtained for a smaller phase angle. In fact, as this angle increases, the illuminated side of the observed object starts facing away from the sensor. The worst illumination condition is obtained when $\phi=180^{\circ}$, meaning that the observed object lies exactly between the sensor and the Sun.

\subsection{Non-Visibility Conditions}

When a space-based telescope is taken into consideration, the limits regarding its ability to continuously track a RSO must also be considered. As a matter of fact, three different configurations can limit the detection capability of the sensor by partially or completely disabling the visibility of the target.

\subsubsection{The Earth Shadow Region}

Depending on the position of the Sun, the shadow casted by the Earth may intersect a portion of the orbit of a spacecraft, promoting the so-called eclipse of the orbit. Since a RSO passing through the shadow of the Earth region is not illuminated by the Sun, such occurrence is considered to be a non-visibility condition for the case of study of this paper.

An approximated method has been used for the evaluation of the eclipse portion of the GEO orbit where the target 
debris, considered during this analysis, moves. The main hypothesis on which this method is based is:

- The Earth is considered as a sphere having a radius of $6371 \mathrm{Km}$.

- The penumbra (partial shadow region placed between the umbra and the full-light region) effects on the eclipse evaluation are ignored.

- The shadow casted by the Earth (umbra) is considered to be a circular cylinder.

Therefore, the intersection between the umbra region and the GEO orbit can be seen as a semi-ellipse whose semiminor axis is equal to the Earth radius $R_{E}$ and semi-major axis is equal to $\frac{R_{E}}{\sin \delta}$ [6], where $\delta$ is the angle between the direction of the Sun rays and the orbital plane. However, in this case of study, since the orbital plane of the GEO orbit lies on the equatorial one, such angle can be considered as the declination of the sun. It is then possible to compute the semi-angular width, $\theta$, of the portion of the GEO orbit lying in the Earth shadow as shown below.

$\theta=\operatorname{acos}\left[\frac{1}{\cos \delta} \sqrt{1-\left(\frac{R_{E}}{r}\right)^{2}}\right]$

Where $r$ is the radius of the GEO orbit: $r=42157 \mathrm{Km}$.

\subsubsection{The Solar Exclusion Region}

The solar exclusion region is defined as the portion of the orbit of the debris, or RSO, where the object lies in a position between the Sun and the sensor. This condition is related to a value of the solar phase angle which is close to $180^{\circ}$, meaning that the observed object appears to be in backlight with respect to the sensor and is therefore impossible to detect. In this work, the solar exclusion region has been considered to have an angular width of $45^{\circ}$ [7].

\subsubsection{The Earth Blockage}

The Earth blockage event occurs when the line of sight of the sensor is blocked by the presence of the Earth along its path.

By assuming a spherical Earth, the computation of the Earth blockage time instants can be trivially reduced to the computation of the time instants when the intersection between a line (the line of sight) and a sphere of radius $R_{E}$ (the Earth) occurs in the Earth-centred intertial reference frame (ECI). In mathematical terms, this is achieved by solving in $\lambda$ the system shown below, in which the ECI location of the sensor at each given time $t$ is identified by the components $\left(X_{\text {sensor }}(t), Y_{\text {sensor }}(t), Z_{\text {sensor }}(t)\right)$ and $\hat{\boldsymbol{u}_{\boldsymbol{X}}}(t), \hat{\boldsymbol{u}_{\boldsymbol{Y}}}(t)$ and $\hat{\boldsymbol{u}_{\boldsymbol{Z}}}(t)$ are the unit vectors of the distance between the sensor and the debris moving along the GEO orbit at each given time.

$\left\{\begin{array}{l}X(t)=X_{\text {sensor }}(t)+\lambda \widehat{\mathbf{u}_{\mathbf{X}}}(t) \\ Y(t)=Y_{\text {sensor }}(t)+\lambda \widehat{\mathbf{u}_{\mathbf{Y}}}(t) \\ Z(t)=Z_{\text {sensor }}(t)+\lambda \widehat{\mathbf{u}_{Z}}(t) \\ X^{2}+Y^{2}+Z^{2}=R_{E}\end{array}\right.$

\section{Numerical Simulation Results on Target Detectability}

\subsection{Simulation Set-up}

The target considered in the first part of the simulation is a $200-\mathrm{cm}$ aluminium diffuse sphere with a reflectance of $18 \%$, composed of a diffuse fraction of $95 \%\left(\rho_{\text {diff }}\right)$ and a specular fraction of 5\% $\left(\rho_{\text {spec }}\right)$ [7]. The simulation has been propagated for a time equal to the orbital period of the target (roughly $24 \mathrm{~h}$ ) during which the sensor, whose orbital period is around $1.5 \mathrm{~h}$, will have completed around 15 orbits. The computation has been carried out for a fixed location of the Sun in the ECI reference frame equal to its vernal equinox position, which means that both declination $\left(\delta_{\text {Sun }}\right)$ and right ascension $\left(\alpha_{\text {Sun }}\right)$ of the Sun are null. The position of the Sun is also used to determine the value of the right ascension of the ascending node (RAAN) of the orbit of the sensor. In order to have a dawn-dusk Sun-synchronous orbit, when the Sun is at the vernal equinox point, such value has to be equal to $270^{\circ}$.

Finally, the location in the ECI reference frame of both sensor and target at the beginning of the simulation (time $t$ $=0$ ) has been chosen to be, respectively, given by the ECI points $\left(0, a_{\text {target }}, 0\right)$ and $\left(a_{\text {sensor }}, 0,0\right)$ where $a_{\text {sensor }}=7001 \mathrm{Km}$ and $a_{\text {target }}=42157 \mathrm{Km}$ are, respectively, the semi-major axis of the orbits of the sensor and the target.

\subsection{Solar Phase Angle and Visual Magnitude Results}

The solar phase angle has been evaluated as follows:

$\phi=a \cos \left(\boldsymbol{u}_{\text {Target }- \text { Sensor }} \cdot \boldsymbol{u}_{\text {Earth }- \text { Sun }}\right)$

where $\boldsymbol{u}_{\text {Target - Sensor }}$ is the target to sensor direction unit vec-

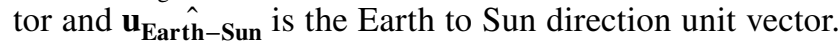
This definition does not match the one given in 2.1 since the direction from the target to the Sun can be approximated with the direction from the Earth to the Sun, based on the fact that the distance between the target and the Earth is negligible if compared to the distance between the Earth and the Sun.

In Fig. 1, it can be noticed that during the description of the first half of the target's orbit, when the target itself gets 


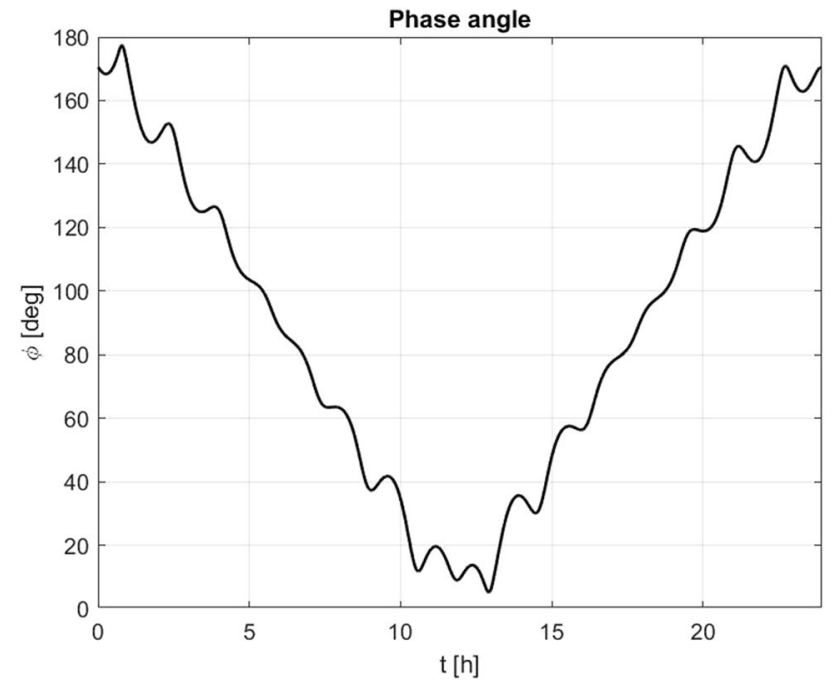

Fig. 1 Phase angle variation for 1 day (orbital period of the target). Sun at vernal equinox

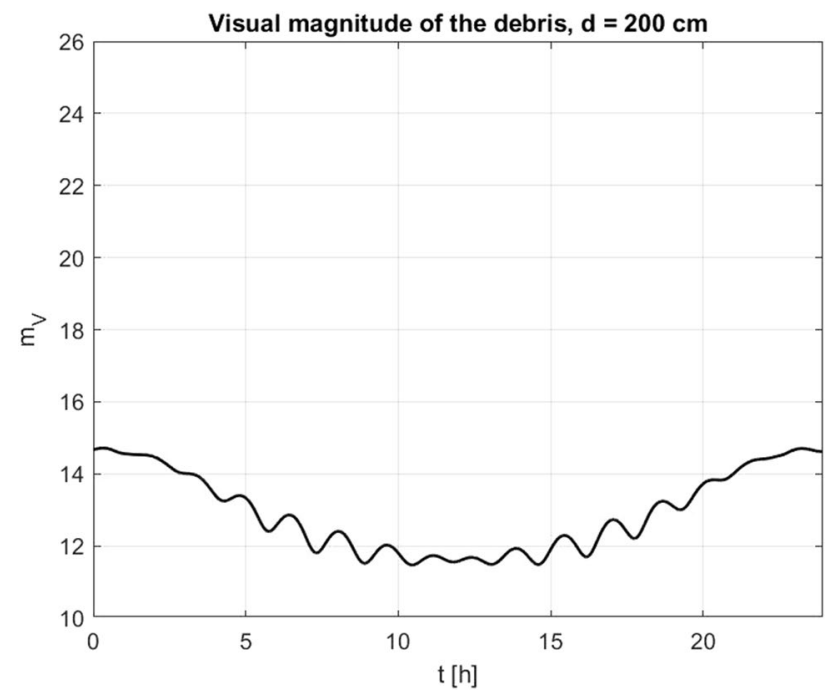

Fig. 2 Visual magnitude variation for 1 day (orbital period of the target). Sun at vernal equinox.

farther from the Sun, the trend of the solar phase angle is a decreasing one while, on the other hand, it gains an increasing trend when the target completes the remaining half of its orbit, getting closer to the Sun. The worst illumination condition is obtained when the target passes through the solar exclusion region with a correspondent "peak" in the visual magnitude of around 15 (Fig. 2). Conversely, the best illumination condition is obtained when the target has completed half of its orbit, thus having its illuminated side facing the sensor. The correspondent visual magnitude shows a constant trend with a mean value of around 11.5 (Fig. 2). Still, this good visibility condition is partially interrupted by the eclipse of the GEO orbit having a duration of $1.15 \mathrm{~h}$. As

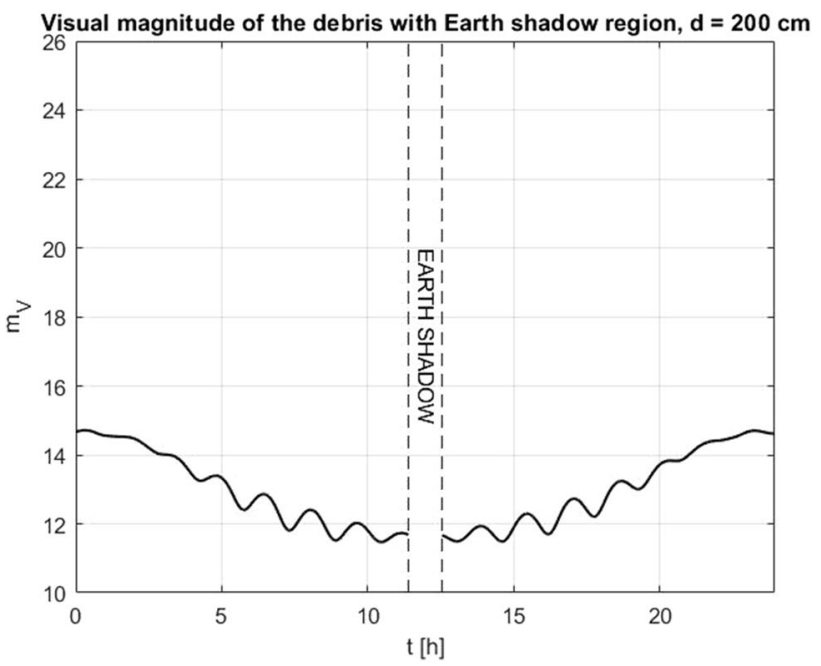

Fig. 3 Visual magnitude variation for 1 day with omitted Earth shadow region. Sun at vernal equinox

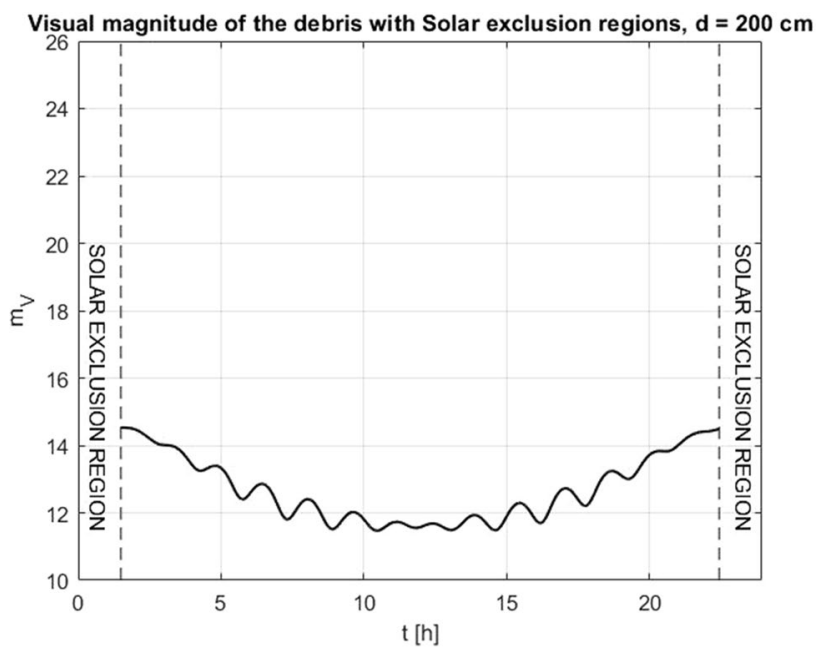

Fig. 4 Visual magnitude variation for 1 day with omitted solar exclusion region. Sun at vernal equinox

shown in Figs. 3 and 4, both the solar exclusion region and the Earth shadow region have been removed from the visual magnitude diagram.

In addition, the computation of the Earth blockage events has shown that, for the given orbit of the sensor, the overall duration of such occurrence is of roughly $11 \mathrm{~h}$ with a total number of distinct events equal to 22. In Fig. 5, each omitted region in the visual magnitude diagram corresponds to a single Earth blockage event.

\subsubsection{Variation in the Diameter of the Target}

Published data on the SBV sensor performances have reported that the sensitivity of such sensor is limited to a 


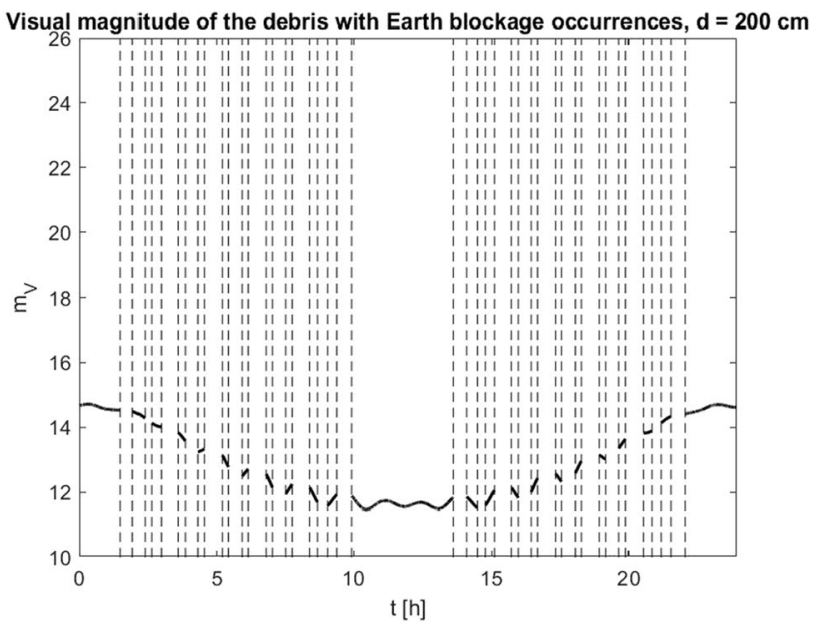

Fig. 5 Visual magnitude variation for 1 day with omitted Earth blockage events. Sun at vernal equinox

visual magnitude of about 15 [7]. In the following results, this value is used to remark the limit in the detection capability of the sensor when the diameter of the observed objects decreases. This is done with the purpose of identifying the minimum detectable size of a debris orbiting in GEO.

In Fig. 6, the green region is intended to be an illustration of the visual magnitude values that the sensor is able to detect as imposed by its limited sensitivity; such region ends with the value $m_{v}=15$. It can easily be seen that a spherical diffuse object with a diameter of $200 \mathrm{~cm}$ is always visible for the chosen sensor. As the diameter decreases, the time interval during which the object appears to be visible decreases too. When the diameter is equal to $50 \mathrm{~cm}$, this time interval is roughly equal to $6 \mathrm{~h}$ while it reaches a null value when the diameter falls off to $30 \mathrm{~cm}$, meaning that the object is never visible. However, the results shown in Fig. 6 do not take in account the non-visibility conditions.

In order to have a clearer idea of the results obtained, an analysis on the fraction of time $\left(t^{*}\right)$ during which the condition $m_{v} \leq 15$ is respected has been performed. A nondimensional parameter $\eta$ has therefore been defined as the ratio between $t^{*}$ and the overall time used for the simulation (orbital period of the target, $T_{\text {target }}$ ). The results of such analysis are shown in Table 1 in terms of the behaviour of $\eta$ when the diameter of the target decreases. In the ideal case, when the non-visibility conditions are not considered (Table 1 top), such parameter follows the results shown in Fig. 6, thus going from unity for a diameter of $200 \mathrm{~cm}$ to the null value for a diameter of $30 \mathrm{~cm}$. However, when the non-visibility conditions are taken into account, as shown in Table 1 bottom, the values of $\eta$ reduce drastically. This is mainly due to the occurrence of the Earth blockage events which do remove more than the $45 \%$ out of the overall time $t^{*}$.

\subsubsection{Variation in the Location of the Sun}

The last analysis carried out concerns the variation in the location of the Sun in the ECI reference frame. The new chosen position is the one corresponding to the summer solstice, when $\alpha_{\text {Sun }}=90^{\circ}$ and $\delta_{\text {Sun }}=23.44^{\circ}$.

Because of the change of position of the Sun, a rotation of the line of nodes of the sensor's orbit has to be considered in order to simulate the dawn-dusk Sun-synchronous condition. For this reason, the value of the RAAN has been changed to $0^{\circ}$. The aforementioned rotation promotes a variation in the initial ECI position of the sensor, which, at the beginning of the simulation, is now given by the components $\left(a_{\text {sensor }}, 0,0\right)$. In addition, a displacement of the solar exclusion region in the ECI reference frame is also obtained, as Fig. 7 witnesses.

By analysing the behaviour of the phase angle in Fig. 8, it is possible to identify the worst illumination condition when the target has roughly completed a quarter of its orbit and is, in fact, passing through the solar exclusion region. As expected, the corresponding visual magnitude reaches a peak of about 14.5 (Fig. 9). The best illumination condition, instead, is obtained when the target lies in the opposite position; the visual magnitude gains a more constant trend with a mean value of around 11.5 (Fig. 8).

By comparing these results with those shown in section 3.2 it has been found that the configuration involving the Sun at the summer solstice is more advantageous since, because of its high declination, the shadow of the Earth does not intercept the equatorial plane where the GEO orbit lies. As a consequence, the target is visible for a longer time.

As shown in Fig. 10, the Earth blockage events disposition in time is changed with respect to that of the previous case. Still, the number of total events and the overall duration are unchanged.

\section{Numerical Simulation Results on Pointing Strategy}

\subsection{The Geosynchronous Pinch Points}

In the following section, the results of the performance analysis of a constellation of two SBV-like sensors operating on the same orbit used in the previous simulations will be discussed. For such sensors, a "fixed intertial" pointing strategy has been adopted; hence, both sensors are kept staring at two different fixed locations in the ECI reference frame with the aim of detecting debris passing through their fields of view.

In order to simulate the maximization of the number of detections, the chosen inertial locations have been considered as those of the pinch points on the GEO belt. These points are characterized by a high density of passing objects, 
Fig. 6 Visual magnitude variation for 1 day (orbital period of the target) for a target having a decreasing diameter from 200 to $30 \mathrm{~cm}$. The green region is an illustration of the visual magnitude range that can be sensed by the sensor. Sun at vernal equinox

both active and inactive, and are visualized in Fig. 11 which shows the population of geosynchronous satellites over a 24-h period [8].

It can easily be seen that the pinch points are both located on the equatorial plane (at a null declination $\delta$ ) and at two different values of right ascension $\alpha$. In the following description, the two points are referred to as "PP1," located at $\alpha_{P P 1}=65^{\circ}$ and "PP2," located at $\alpha_{P P 2}=245^{\circ}$.

\subsubsection{Genesis of the Pinch Points}

The pinch points are the consequence of different factors among which are the particular type of orbit and its evolution in time and the common strategy typically applied by geosynchronous (GS) and geostationary satellites operators.

During its lifetime, a GS satellite faces an out-of-plane force caused by the action of the perturbation promoted by the Sun and the Moon (also referred to as lunisolar perturbation), and the perturbation caused by the oblateness of the Earth. This force causes a periodic variation in the inclination and RAAN of the orbit on which the satellite operates. As shown in Fig. 12, it takes 27 years for the orbit's inclination of a GS satellite to increase up to $15^{\circ}$ and nearly the same time for it to get back to its initial value. During this variation, the RAAN evolves from $+90^{\circ}$ to $-90^{\circ}$.

The evolution pattern shown in Fig. 12 is followed by the majority of the inactive GS satellites which are no longer manoeuvred as to keep their orbit's inclination as close as possible to $0^{\circ}$, thus creating a geostationary satellite. GS satellites operators choose to launch their satellites with an initial value of inclination and RAAN that places the evolution of the satellite along the curve in Fig. 12.

It is the joint effect of the natural perturbations and the choice of initial conditions decided by the operators that promotes the creation of the pinch points congestion regions.

\subsection{Simulation Set-up}

The illustration in Fig. 13 shows the initial geometry chosen for the simulation, where the first sensor (S1) is located at the ascending node of the orbit and the second sensor (S2) is located in the opposite position. In particular, sensor $\mathrm{S} 1$ is pointed towards the pinch point $\mathrm{PP} 1$ and sensor $\mathrm{S} 2$ is pointed toward the pinch point PP2. In this case, the Sun is considered as fixed at the vernal equinox point.

The analysis of the performance deals with the capability of each sensor to detect targets (debris) distributed on the GEO orbit during a period of $24 \mathrm{~h}$. For this reason, the
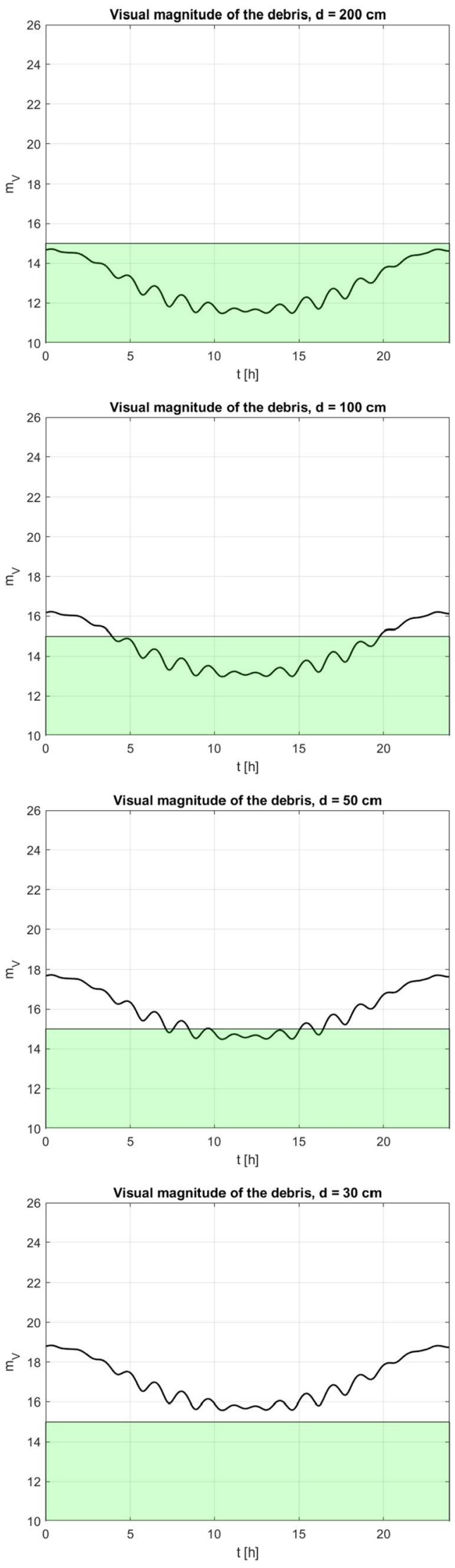

sensor in terms of values of the adimensional parameter $\eta$. Non-visibility conditions not considered (top), non-visibility conditions considered (bottom)
Table 1 Peformances of the

\begin{tabular}{ll}
\hline Diameter [cm] & $\eta=\frac{t^{*}}{T_{\text {target }}}$ \\
\hline 200 & 1.0 \\
150 & 0.81 \\
100 & 0.66 \\
50 & 0.31 \\
40 & 0.028 \\
30 & 0 \\
200 & 0.22 \\
150 & 0.39 \\
100 & 0.10 \\
50 & 0.050 \\
40 & 0.020 \\
30 & 0 \\
\hline
\end{tabular}

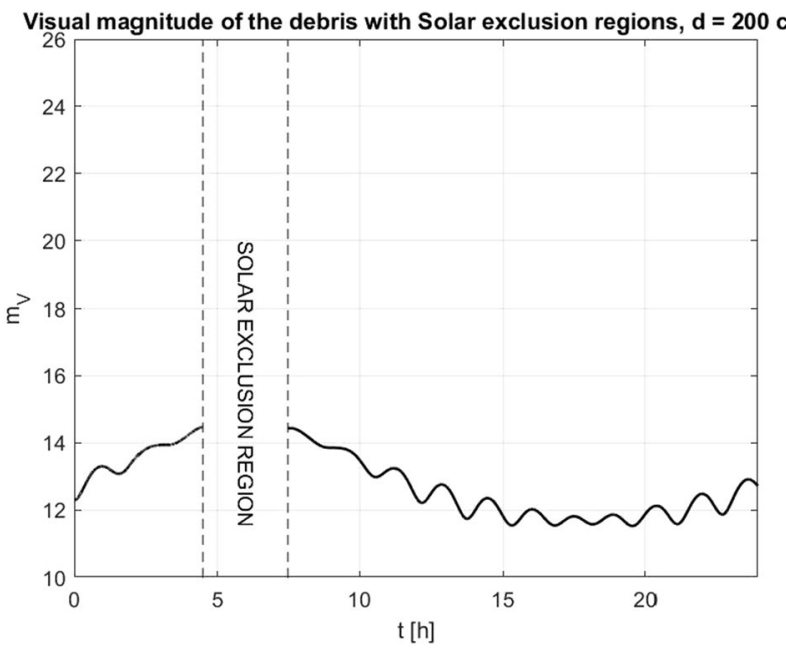

Fig. 7 Visual magnitude for 1 day (orbital period of the target) with omitted solar exclusion region. Sun at summer solstice

simulation includes an ideal distribution of targets in GEO, whose position is presented in Fig. 14. Such position is listed in Table 2 in terms of the true anomaly $v$ of each target at the beginning of the simulation. For the sake of clarity it has to be noticed that for a circular orbit like the GEO one, the definition of a true anomaly angle loses its meaning. Nevertheless, it has been used during this study as to give an immediate idea of the position of an object along its orbit. The null value of $v$ along the GEO orbit has been set on the point of intersection between the ECI positive $\mathrm{X}$ axis and the orbital plane.

For the detection to occur, the target must cross the FoV of one of the sensors, which has an angular width of $1.4^{\circ}$ centred around the location of the observed pinch point. It is then considered that the target crosses the FoV of the sensor when the angle between the direction of the line of sight of the sensor (sensor to pinch point direction) and the sensor

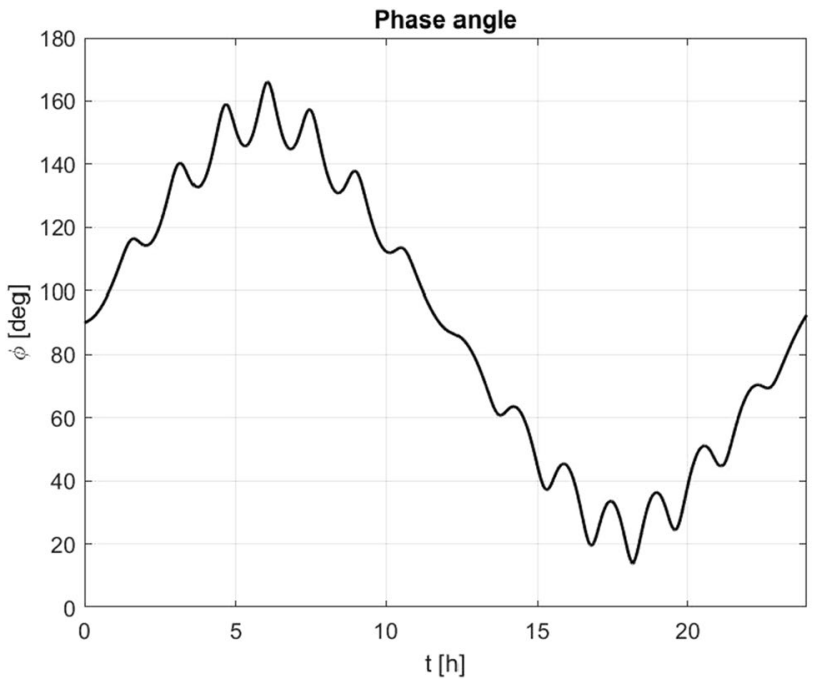

Fig. 8 Phase angle variation for 1 day (orbital period of the target). Sun at summer solstice

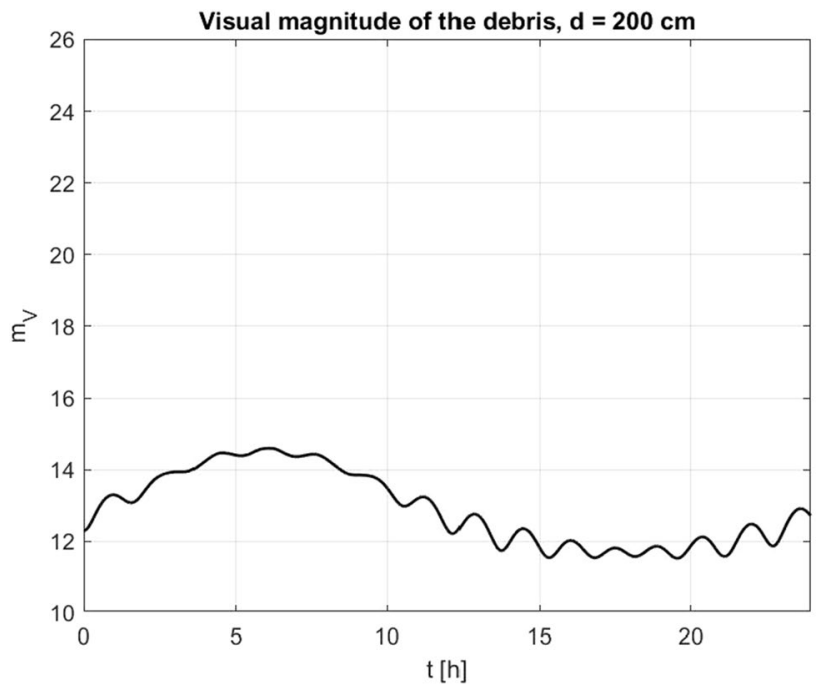

Fig. 9 Visual magnitude for 1 day (orbital period of the target). Sun at summer solstice

to target direction is smaller than half of the angular width of the FoV $\left(0.7^{\circ}\right)$.

It has been found that the pinch points do not fall within any of the non-visibility regions; hence, the only limit affecting the detection of a specific target is the occurrence of an Earth blockage during the crossing of the target in the sensor's FoV.

\subsection{Performance results}

The performance of the constellation is mainly analysed in terms of number of detections achieved by each sensor and duration of each observation. 


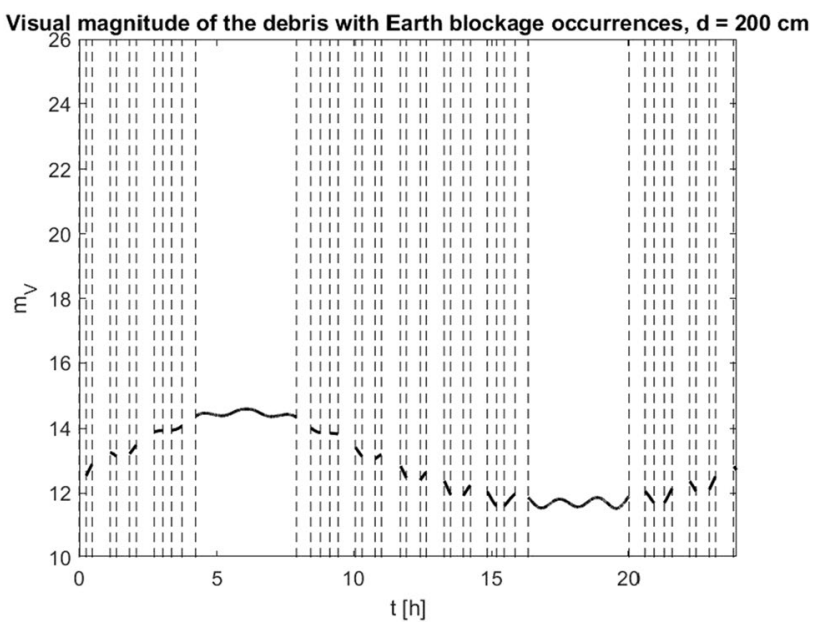

Fig. 10 Visual magnitude for 1 day (orbital period of the target) with omitted Earth blockage events. Sun at summer solstice

In Table 3, the observation duration is reported. This quantity has been evaluated as the difference between the time instant when a certain target is last "seen" by the sensor and the time instant when it is first seen by the same. Because of the occurrence of the Earth blockage, this duration is typically different for every target. In the worst scenario, the Earth blockage completely blocks the detection of a target causing a null value of the observation duration.

It is easy to understand how advantageous the use of a constellation of two sensors is in terms of number and duration of each observation. As a matter of fact, the performance of sensor $\mathrm{S} 1$, which is only able to detect 3 out of 9 targets, two of which are observed for less than one minute, is far to be as satisfactory as the one of sensor S2. This latter sensor is, on the other hand, able to observe 7

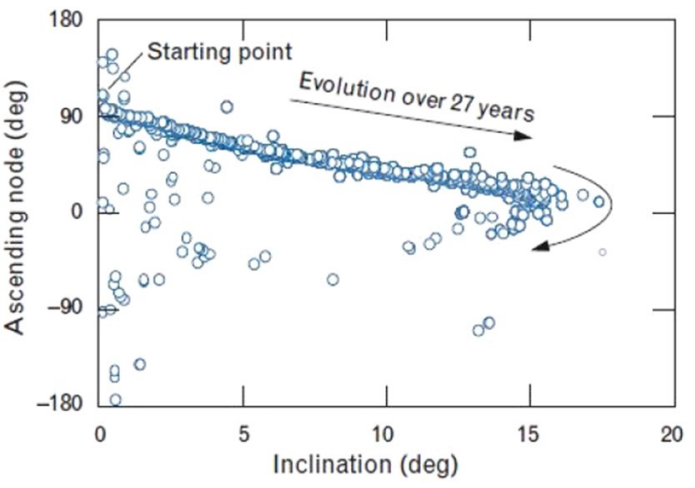

Fig. 12 Evolution of the inclination and RAAN of geosynchronous satellites (blue dots) caused by the lunisolar and $J_{2}$ perturbations. Reprinted with permission Courtesy of MIT Lincoln Laboratory, Lexington, Massachusetts [8]

out of 9 targets, five of which are observed for a time that goes from three minutes to almost six minutes.

By using a constellation of sensors not only can we ensure a complementarity in the observation of the targets, but also a greater number of observations (thus further measurements) of the same target.

\subsubsection{Variation in the Position of the Sun}

When the Sun is located at the summer solstice point, the rotation of the line of nodes of the orbit of the sensors in the ECI reference frame causes a different temporal disposition of the Earth blockage events, as shown in Fig. 10. For this reason, a change in the observation durations of the constellation is expected.

Still, the different ECI location of the solar exclusion region does not interfere with the detection of any target since none of the observed space windows (pinch points) fall
Fig. 11 Illustration of the population of satellites on the geosynchronous belt in a 24-h period as seen in the ECI reference frame. The contours show the number of objects passing through a $1.4^{\circ} \times 1.4^{\circ}$ FoV. Reprinted with permission Courtesy of MIT Lincoln Laboratory, Lexington, Massachusetts. [8]

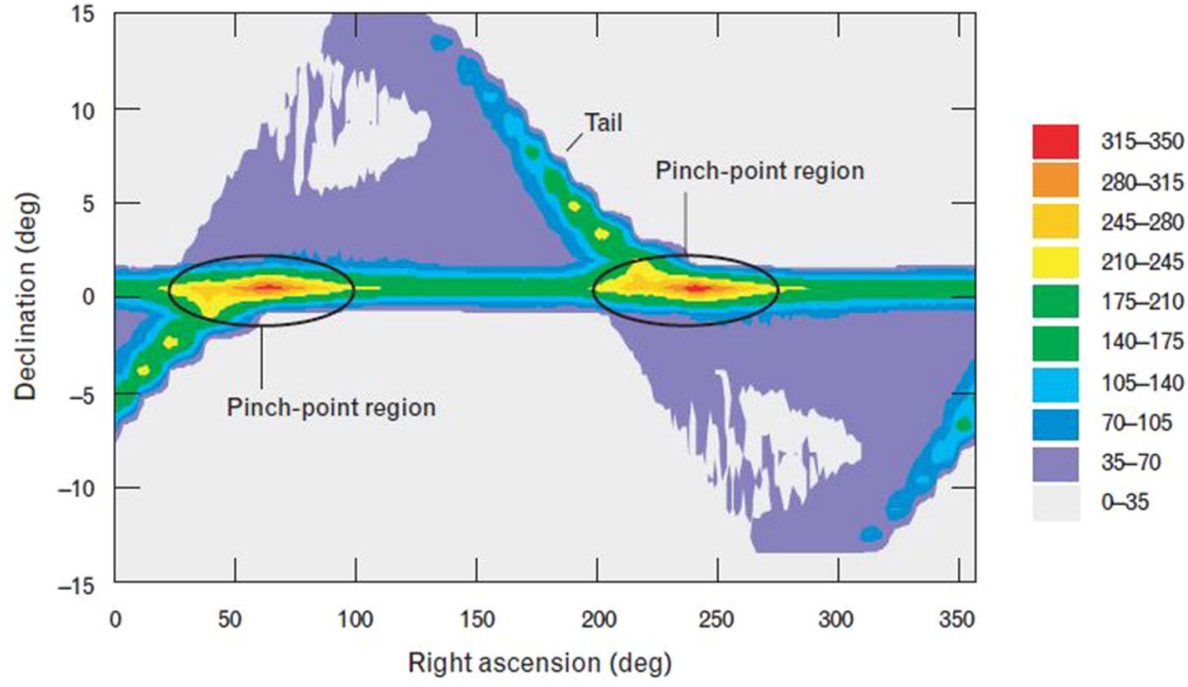


Fig. 13 MATLAB illustration of the geometry at the beginning of the simulation. The line of sights (red lines) of the two sensors (black dots) are pointed towards the pinch points

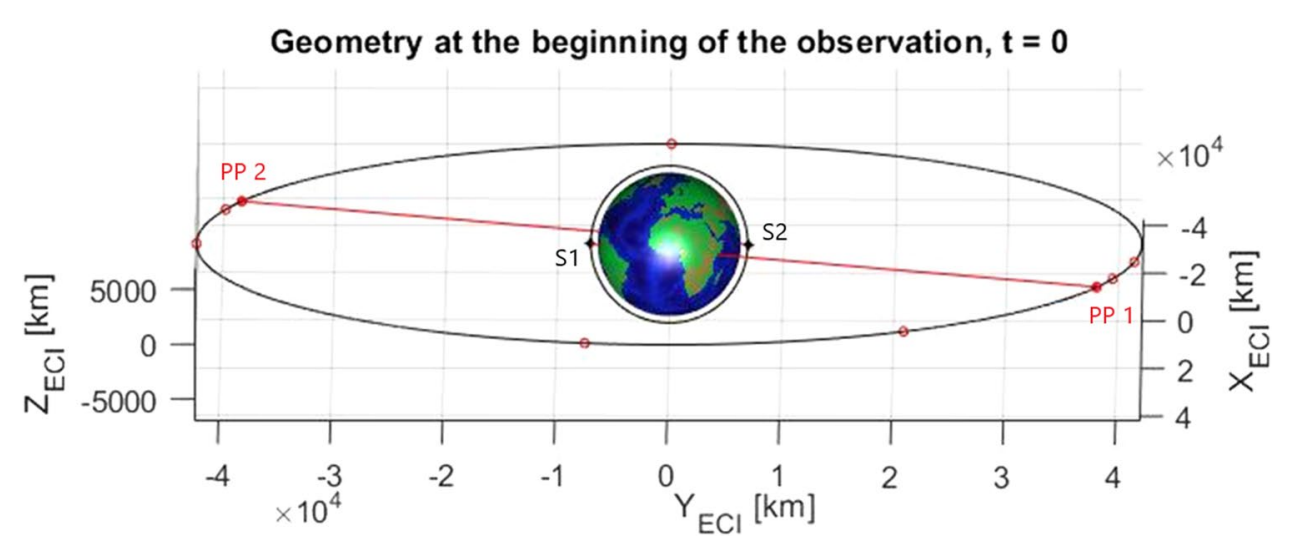

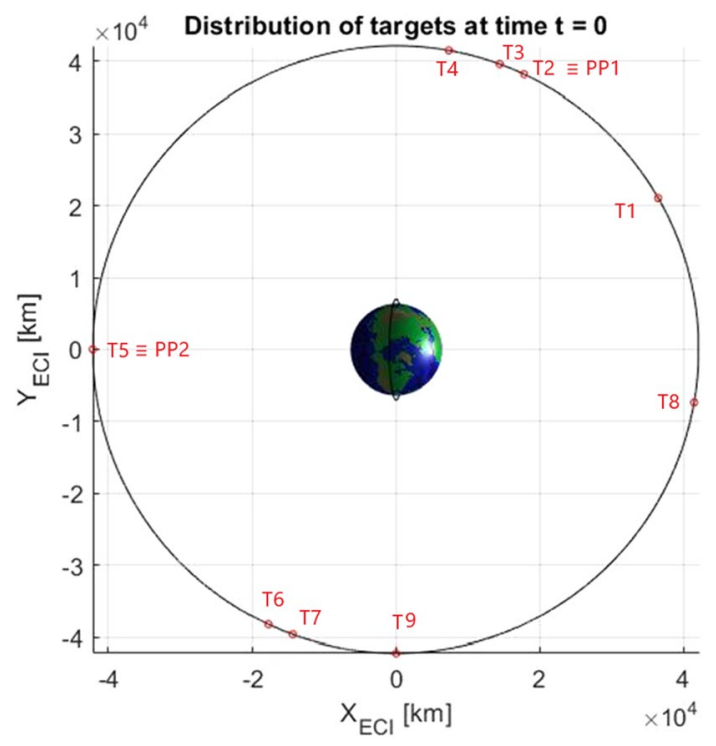

Fig. 14 MATLAB illustration of the distribution of targets (red dots) at time $t=0$
Table 2 Location of targets along their orbit at time $t=0$ in terms of true anomaly

\begin{tabular}{ll}
\hline Target & $v_{0}$ \\
\hline T1 & $30^{\circ}$ \\
T2 & $65^{\circ}$ \\
T3 & $70^{\circ}$ \\
T4 & $80^{\circ}$ \\
T5 & $245^{\circ}$ \\
T6 & $250^{\circ}$ \\
T7 & $270^{\circ}$ \\
T8 & $350^{\circ}$ \\
T9 & $180^{\circ}$ \\
\hline
\end{tabular}

within such region. The Earth blockage is, again, the only limit for the detection of targets to be achieved.

As Table 4 shows, the performance of sensor $\mathrm{S} 1$ is now improved with respect to the one of the previous cases. In
Table 3 Performance of sensors S1 (top) and S2 (bottom). Sun at vernal equinox

\begin{tabular}{ll}
\hline Target & $\begin{array}{l}\text { Observation } \\
\text { duration } \\
{[\text { min] }}\end{array}$ \\
\hline T1 & 0 \\
T2 & 0 \\
T3 & 0 \\
T4 & 0 \\
T5 & 0.15 \\
T6 & 0.12 \\
T7 & 2.8 \\
T8 & 0 \\
T9 & 0 \\
T1 & 1.4 \\
T2 & 0.15 \\
T3 & 0.12 \\
T4 & 5.7 \\
T5 & 2.8 \\
T6 & 0 \\
T7 & 5.6 \\
T8 & 0 \\
T9 & 3.7 \\
\hline
\end{tabular}

fact, the sensor is now able to detect 6 out of 9 targets, four of which are also observed for the whole duration of their transit through the FoV of the sensor. The performance of this sensor is now comparable with that of sensor S2 which does still show a greater productivity, being it able to detect 7 out of 9 targets.

It appears to be clear that an improvement in the overall performance of the constellation is obtained when the Sun is located at the summer solstice since the Earth blockage events have been found to occur less frequently during the passage of targets in the FoV of the sensors. 
Table 4 Performance of sensors S1 (top) and S2 (bottom). Sun at summer solstice

\begin{tabular}{ll}
\hline Target & $\begin{array}{l}\text { Observation } \\
\text { duration } \\
\text { [min] }\end{array}$ \\
\hline T1 & 0 \\
T2 & 2.7 and 2.8 \\
T3 & 0.3 \\
T4 & 5.5 \\
T5 & 0 \\
T6 & 5.6 \\
T7 & 0 \\
T8 & 5.3 \\
T9 & 5.4 \\
T1 & 5.4 \\
T2 & 0 \\
T3 & 5.6 \\
T4 & 5.5 \\
T5 & 2.7 and 2.8 \\
T6 & 0.3 \\
T7 & 5.5 \\
T8 & 0 \\
T9 & 3.7 \\
\hline
\end{tabular}

However, a clear advantage in the implementation of a space-based telescope in a space surveillance network is found when the goal of its mission is the detection of GEO debris. As a matter of fact, as the last simulation has shown, in a 24-h period, such sensor is ideally able to detect many objects orbiting in the GEO ring thus having a quick access to this particular space region. A ground-based telescope pointed towards GEO, because of the peculiarity of this type of orbit, would only be able to continuously follow those debris which fall in its FoV.

The last simulation of this study has been developed as to identify the possible advantage of the use of a constellation of two SBV-like sensors operating on the same orbit. Such constellation was kept "staring" at a fixed region in the inertial space which was chosen to coincide with the geosynchronous pinch points region. Thanks to the inertial location of such points in addition to the two positions of the Sun, the two sensors do not experience the constrain given by the Earth shadow and the solar exclusion region. The performances, studied in terms of the duration of the observation of each target and number of detected targets, showed that, as expected, the use of a constellation is a clear advantage in terms of productivity.

Funding Open access funding provided by Università degli Studi di Napoli Federico II within the CRUI-CARE Agreement..

Open Access This article is licensed under a Creative Commons Attribution 4.0 International License, which permits use, sharing, adaptation, distribution and reproduction in any medium or format, as long as you give appropriate credit to the original author(s) and the source, provide a link to the Creative Commons licence, and indicate if changes were made. The images or other third party material in this article are included in the article's Creative Commons licence, unless indicated otherwise in a credit line to the material. If material is not included in the article's Creative Commons licence and your intended use is not permitted by statutory regulation or exceeds the permitted use, you will need to obtain permission directly from the copyright holder. To view a copy of this licence, visit http://creativecommons.org/licenses/by/4.0/.

\section{References}

1. ESA Space Debris Office, ESA's Annual Space Environment Report (2019)

2. Bonnal, C., McKnight, D.S.: IAA Situational Report on Space Debris-2016 (2016)

3. Harrison, D.C., Chow, J.C.: The space-based visible sensor. John Hopkins Apl. Techn. Dig. 17, 226-236 (1996)

4. Shell, J.R.: Commercially-Hosted Payloads for Debris Monitoring and Mission Assurance in GEO, (2011)

5. Kervin, P.W., Hall, D., Bolden, M., Toth, J.: Phase Angle: What is it good for?. Advanced Maui Optical and Space Surveillance Conference, Maui, Hawaii (2010)

6. de Iaco, V.A.: Shadow times of earth satellites. Rivista Italiana di Compositi e Nanotecnologie. Springer, Berlin (2014)

7. Ackermann, R.M., Colonel Kiziah, R.R., Zimmer, P.C., McGraw, J.T., Cox, D.D.: A Systematic Examination of Ground-Based and 
Space-Based Aprroaches to Optical Detection and Tracking of Satellites, 31st Space Symposium. Colorado Springs, Colorado (2015)

8. Sharma, J., Stokes, G.H., von Braun, C., Zollinger, G., Wiseman, A.J.: Toward operational space-based space surveillance. Linc. Lab. J. 13, 309-334 (2002)
Publisher's Note Springer Nature remains neutral with regard to jurisdictional claims in published maps and institutional affiliations. 